\title{
Ações de saúde para homens-pais e a promoção à paternidade no pré-natal: Revisão integrativa
}

\author{
Health actions for men-fathers and promotion of fatherhood in prenatal care: Integrative review \\ Acciones de salud para hombres-padres y la promoción de la paternidad en la atención prenatal: \\ Una revisión integradora
}

Recebido: 30/03/2021 | Revisado: 09/04/2021 | Aceito: 13/04/2021 | Publicado: 24/04/2021

Bruna Celia Lima de Oliveira

ORCID: https://orcid.org/0000-0002-1468-5552

Universidade Federal do Estado do Rio de Janeiro, Brasil

E-mail: limabrucs@gmail.com

Amilton Douglas Ferreira de Araújo

ORCID: https://orcid.org/0000-0002-3681-8053

Federal do Estado do Rio de Janeiro, Brasil

E-mail: amilton.douglas@gmail.com

Mayara Ribeiro Maciel

ORCID: https://orcid.org/0000-0002-8454-9521

Universidade Federal do Estado do Rio de Janeiro, Brasil

E-mail: mrmenf@gmail.com

Bianca Pezzini Souza da Silva Klayn

ORCID: https://orcid.org/0000-0002-9478-4605

Universidade Federal Fluminense, Brasi

E-mail: biancapezzini@yahoo.com.br

Cláudia Regina Ribeiro

ORCID: https://orcid.org/0000-0002-0262-0073 Universidade Federal Fluminense, Brasil

E-mail: 13cribeiro@gmail.com

Adriana Lemos

ORCID https://orcid.org/0000-0001-9705-6200

Universidade Federal do Estado do Rio de Janeiro, Brasil

E-mail: adrianalemos@unirio.br

\begin{abstract}
Resumo
Objetivo: conhecer a produção científica sobre as ações de cuidado à saúde e promoção à paternidade para homens-pais durante o período pré-natal, em âmbito nacional e internacional. Método: revisão integrativa realizada nas bases de dados CINAHL, SCOPUS, LILACS e MEDLINE. A questão norteadora do estudo seguiu a estratégia PICo: O que as produções científicas dizem a respeito das ações de cuidado à saúde dos homens-pais e a promoção da paternidade durante o período pré-natal? Os critérios de inclusão: artigos científicos completos, publicados nas línguas português, inglês e espanhol, que abordassem a temática ações de cuidado à saúde para homens-pais e promoção da paternidade com acesso online e de forma gratuita, entre os anos de 2010 a agosto de 2020. Critérios de exclusão: literatura cinzenta, artigos duplicados ou que não contemplassem a temática da pesquisa. Resultados: os estudos apontam que as ações de saúde para os homens estão concentradas no planejamento reprodutivo, especificamente na obtenção de métodos contraceptivos e também no rastreio de infecções sexualmente transmissíveis. Ressalta-se que o homem-pai ainda é coadjuvante do cuidado, com foco no binômio mãe-bebê. Conclusão: as produções científicas mostram que os homenspais estão em maioria nas ações de planejamento reprodutivo, mas não há uma articulação com o pré-natal do parceiro, e cuidados à sua saúde. Dificuldades e estratégias para inclusão desta população são elencadas nos estudos.
\end{abstract}

Palavras-chave: Cuidado pré-natal; Paternidade; Saúde do homem.

\begin{abstract}
Objective: to know the scientific production on health care actions and promotion of fatherhood for men-fathers during the prenatal period, nationally and internationally. Method: integrative review conducted in the CINAHL, SCOPUS, LILACS and MEDLINE databases. The guiding question of the study followed the PICo strategy: What do scientific productions say about health care actions for men-fathers and the promotion of fatherhood during the prenatal period? Inclusion criteria: complete scientific articles, published in Portuguese, English, and Spanish, that addressed the theme of health care actions for men-fathers and the promotion of fatherhood with online and free access, between the years 2010 and August 2020. Exclusion criteria: gray literature, duplicate articles, or articles that didn't address the research theme. Results: the studies point out that health actions for men are focused on reproductive planning, specifically in
\end{abstract}


obtaining contraceptive methods and also in screening for sexually transmitted infections. It is emphasized that the manfather is still a coadjuvant of care, focusing on the mother-baby binomial. Conclusion: The scientific productions report that men-fathers are in majority in the actions of reproductive planning, but there is no articulation with the prenatal of the partner, and care for his health. Difficulties and strategies for inclusion of this population are listed in the studies.

Keywords: Prenatal care; Paternity; Men's health.

\section{Resumen}

Objetivo: conocer la producción científica sobre las acciones de cuidado de la salud y promoción de la paternidad para los hombres-padres durante el período prenatal, en el ámbito nacional e internacional. Método: revisión integradora realizada en las bases de datos CINAHL, SCOPUS, LILACS y MEDLINE. La pregunta guía del estudio siguió la estrategia PICo: ¿Qué dicen las producciones científicas sobre las acciones de atención a la salud de los hombres-padres y la promoción de la paternidad durante el período prenatal? Criterios de inclusión: artículos científicos completos, publicados en portugués, inglés y español, que aborden el tema de las acciones de atención a la salud de los hombrespadres y la promoción de la paternidad con acceso online y gratuito, entre los años 2010 y agosto de 2020. Criterios de exclusión: literatura gris, artículos duplicados o que no contemplaran el tema de la investigación. Resultados: los estudios apuntan a que las acciones de salud para hombres se concentran en el plano reproductivo, específicamente en la obtención de métodos anticonceptivos y también en el rastreo de infecciones sexualmente transmisibles. El hombrepadre sigue siendo un coadyuvante de los cuidados, centrándose en el binomio madre-bebé. Conclusión: los resultados científicos relatan que los hombres-padres se encuentran en su mayoría en las acciones de planificación reproductiva, pero no hay una articulación con el prenatal del parceiro, y los cuidados a su salud. En los estudios se enumeran las dificultades y estrategias para la inclusión de esta población.

Palabras clave: Atención prenatal; Paternidad; Salud del hombre.

\section{Introdução}

A Política Nacional de Atenção Integral à Saúde do Homem (PNAISH) tem como objetivo facilitar e ampliar o acesso da população masculina às ações e serviços, sobretudo os da atenção básica, sob a perspectiva relacional de gênero e na lógica da concepção de linhas de cuidado que respeitem a integralidade da atenção. Desse modo, visa contribuir de forma efetiva para a redução da morbimortalidade masculina no Brasil (Brasil, 2008).

Além de tratar tais questões, a PNAISH aposta na perspectiva de inclusão do tema paternidade e cuidado a partir da promoção da saúde sexual e reprodutiva. Sendo assim, alguns municípios brasileiros desenvolveram estratégias que visam a valorização da paternidade a partir da inclusão dos homens-pais no pré-natal de suas companheiras (Ribeiro, Gomes \& Moreira, 2017).

De forma especial, o Ministério da Saúde em 2016 lançou o Guia de Saúde do Homem para Agentes Comunitários (ACS), que aborda esses temas e, no mesmo ano, o Guia do Pré-Natal do Parceiro para Profissionais de Saúde, que tem como foco ações voltadas para o planejamento reprodutivo como uma estratégia essencial para qualificar a atenção à gestação, parto e ao nascimento a partir da valorização da participação dos homens. Logo, o homem é envolvido ativamente no processo de planejamento reprodutivo, viabilizando-o como sujeito desse cuidado (Hermann, 2016).

Além da PNAISH e dos Guias, iniciativas do Ministério da Saúde tais como a estratégia Rede Cegonha (Brasil, 2011), e a Lei $n^{\circ} 11.108$ de 7 de abril de 2005 (Brasil, 2005), conhecida como lei do acompanhante, trazem a garantia da presença de um/a acompanhante no trabalho de parto, parto e pós-parto, também contribuindo positivamente para o incentivo à inserção dos homens ainda nas consultas de pré-natal. Mudanças que colaboram para o questionamento do paradigmático binômio mãe-bebê ao dar lugar ao trinômio pai-mãe-bebê.

Portanto, as estratégias que visam a inclusão dos homens no pré-natal do parceiro e/ou pré-natal masculino - constituemse como uma "porta de entrada positiva" para os homens no serviço de saúde, aproveitando sua presença nas consultas para realizar os testes rápidos, os convidando a participar das atividades educativas que estimulam o exercício da paternidade e, muito importante, ofertando a eles cuidados de saúde que extrapolam a saúde da mãe e do bebê, buscando a integralidade do cuidado ao tratá-los como sujeitos de direito em saúde e não como um possível infectante dessa díade (Ribeiro, Gomes \& Moreira, 2017). 
No entanto, apesar das políticas ministeriais, são poucos os municípios que vêm desenvolvendo ações voltadas para os homens e os homens-pais fora do âmbito do Novembro Azul, sendo ainda pouca a presença dessa população nos serviços de atenção básica e nas atividades do pré-natal (Nascimento, Marcelino, Vieira \& Lemos, 2019).

Logo, os homens-pais estando presentes nas consultas de pré-natal é uma oportunidade para este estar acessando os serviços de atenção básica, de forma de tenha maior aproximação com as ações de promoção à sua saúde de forma integral. Além do estímulo e trabalho com as questões relacionadas à paternidade e cuidado.

Destaca-se a ideia de "resolver o problema" ou "ficar bom logo", colocando a ênfase na ação curativa e não na prevenção e promoção à saúde. Ao qual tem uma íntima relação com as atribuições destinadas socialmente aos homens: serem trabalhadores e provedores da família. Esse pensamento atravessa e organiza as práticas de assistência, corroborando na construção da ideia de uma restituição rápida da força física masculina, a sua força de trabalho. Isto leva os homens a estarem mais presentes nos serviços de emergência e nos hospitais, do que de fato com a atenção primária, em práticas de promoção à saúde (Schraiber \& Figueredo, 2011).

Dessa forma, há uma necessidade de conhecer as produções científicas não só em contexto brasileiro, mas mundial, e como estas estão realizando as ações de saúde para homens-pais durante o período pré-natal e a promoção da paternidade neste período. Nessa perspectiva surge a inquietação para esta pesquisa, partindo do seu objetivo, ao qual é conhecer a produção científica sobre as ações de cuidado à saúde e promoção à paternidade para homens-pais durante o período pré-natal, em âmbito nacional e internacional.

\section{Metodologia}

A revisão integrativa foi escolhida como método desta pesquisa. Esta é uma ferramenta que sintetiza as pesquisas disponíveis em um determinado tema e direciona a prática fundamentando-a no conhecimento científico disponível (Souza, Silva \& Carvalho, 2010). Portanto, possibilita um melhor aperfeiçoamento do conhecimento sobre uma temática, avaliando as evidências disponíveis, proporcionando a junção do conhecimento sobre o assunto, além de identificar lacunas de aspectos que precisam ser mais aprofundados (Mendes, Silveira \& Galvão, 2008).

A revisão integrativa é intitulada a mais ampla abordagem metodológica das revisões, pois permite o acréscimo de estudos experimentais e não experimentais, fazendo uma junção rigorosa e sistematizada do estudo sobre o fenômeno de investigação (Souza et al., 2010). Além disso, combina dados da literatura teórica e empírica, contemplando diversos propósitos: definição de conceitos, revisão de teorias e evidências e análise de problemas metodológicos de uma especificidade. A ampla amostra em conjunto com a multiplicidade de propostas, resulta em um panorama sólido e compreensível de conceitos complexos, teorias ou problemas de saúde relevante principalmente para a área da enfermagem (Whittermore \& Knafl, 2005)

Esta revisão integrativa cumpriu as seguintes etapas: $1^{\circ}$ Definição da pergunta da pesquisa; $2^{\circ}$ Busca ou amostragem na literatura - critérios de inclusão e exclusão de estudos -; $3^{\circ}$ Coleta de dados - definição do documento para reunir e sintetizar as informações; categorização dos estudos; $4^{\circ}$ avaliação dos estudos aos quais foram incluídos na revisão - avaliar o nível de evidência dos estudos; $5^{\circ}$ Interpretação dos resultados do estudo - discussão dos resultados com o conhecimento teórico identificação de conclusões e implicações resultantes; $6^{\circ}$ apresentação da revisão integrativa - elaboração do documento para descrição das etapas utilizadas. (Souza et al., 2010; Mendes et al., 2008).

Este estudo foi realizado por seis revisores independentes. Para auxílio à questão norteadora da pesquisa, realizou-se a estratégia PICo. A estratégia tem como fundamento a Pesquisa Baseada em Evidência, ao qual decompõe e caracteriza os problemas clínicos que emergem na pesquisa, assistência ou ensino. Os elementos da estratégia PICO são descritos: P, pessoa ou população; I, intervenção a ser considerada; C, comparação; O, outcomes, desfecho/ resultado (Santos, Pimenta \& Nobre, 
2007). Ressalta-se que embora este modelo seja bastante utilizado, nem sempre se adapta aos múltiplos contextos da área da saúde, portanto este sofreu algumas modificações em modelos alternativos (Sousa et al., 2008).

Nesse sentido, foi construído para esta pesquisa os acrônimos da estratégia PICo, onde P: população - homens-pais -; I: fenômeno de interesse - ações de cuidado à saúde e promoção a paternidade -; Co: contexto - período pré-natal -. Formulandose a seguinte questão norteadora: $\mathrm{O}$ que as produções científicas dizem a respeito das ações de cuidado à saúde dos homenspais e a promoção da paternidade durante o período pré-natal?

A partir disso, foi realizada a busca estratégica durante o mês de agosto do ano de 2020. Para a escolha dos descritores, utilizaram-se os Descritores em Ciência e Saúde (DeCs), bem como a leitura dos conceitos destas palavras-chave para composição: Cuidado Pré-natal; Paternidade; Saúde do Homem. Consultou-se os Descritores (DeCS/MeSH) e foram definidas as terminologias: - Paternidade/Paternity -; Cuidado Pré-natal/Prenatal Care -; - Saúde do Homem/Men 's Health -.

Para manter a coerência e ampliar a busca, utilizou-se como estratégia o cruzamento entre os termos, usando o operador lógico booleano and, gerando as seguintes combinações: "Paternidade and Saúde do Homem" "Paternity and Men's Health", "Paternidade and Cuidado Pré-natal" "Paternity and Prenatal Care", "Paternidade and Cuidado Pré-natal and Saúde do Homem" "Paternity and Prenatal Care And Men's Health".

Realizou-se a busca em quatro bases de dados: CINAHL - Cumulative Index to Nursing and Allied Health Literature -; SCOPUS - SciVerse Scopus -; LILACS - Literatura Latino-americana e do Caribe em Ciências da Saúde -; MEDLINE - Medical Literature Analysis and Retrieval System Online.

Os critérios de inclusão: artigos científicos completos, publicados nas línguas português, inglês e espanhol, que abordassem a temática ações de cuidado à saúde para homens-pais e promoção da paternidade com acesso online e de forma gratuita. Publicados entre o recorte temporal de 2010 a agosto de 2020. Optou-se por este recorte devido a Política Nacional de Atenção Integral à Saúde do Homem em 2009 e o Guia Pré-Natal do Parceiro para Profissionais de Saúde em 2016.

Os critérios de exclusão: literatura cinzenta (teses, dissertações e livros), artigos duplicados ou que não contemplassem a temática da pesquisa. Foi elaborado um questionário pelas autoras contendo os seguintes itens: Identificação do estudo, objetivo, metodologia, resultado e nível de evidência, tal como propõe Souza et al (2010).

A fim de realizar uma avaliação dos estudos encontrados, primeiramente os autores leram o título, resumo e palavraschave dos artigos, com intuito de identificar uma coerência dos artigos com a questão norteadora da pesquisa e organizar os artigos pré-selecionados. Os artigos pré-selecionados foram lidos de forma integral e avaliados quanto à elegibilidade para a pesquisa.

Para a avaliação do nível de evidência, utilizou-se uma hierarquização das evidências, segundo o delineamento dos artigos selecionados, aos quais são descritos: Nível 1- evidências resultantes da meta-análise de múltiplos estudos clínicos e controlados e randomizados - ; Nível 2: - evidências obtidas em estudos individuais com delineamento experimental -; Nível 3 evidências de estudos quase experimentais - ; Nível 4:- evidências de estudos descritivos (não experimentais) ou com abordagem qualitativa -; Nível 5 - evidências provenientes de relatos de caso ou de experiência -; Nível 6 - evidências baseadas em opiniões de especialistas (Stetler, Morsi \& Rucki, 1987).

A Prática Baseada em Evidências estimula a utilização dos resultados de pesquisa na assistência à saúde, na qual a revisão integrativa é um método utilizado na prática baseada em evidências, em que possibilita a utilização das evidências na prática clínica (Dalfolvo, Lana \& Silveira, 2008).

Após essas fases terminadas, pode-se prosseguir com a revisão integrativa na discussão dos resultados, a qual ocorreu a análise dos dados, sua apreciação e resumo conforme o suporte teórico preconizado, e assim a apresentação da revisão integrativa, que teve a intenção de ser mais clara possível, e não permitindo ambiguidade ou incoerências, propiciando ao leitor aprender os resultados da pesquisa de forma crítica (Souza et al.,2010). 


\section{Resultados}

Foram encontrados 49 artigos nas bases de dados, sendo 1 na CINAHL, 12 na SCOPUS, 16 na LILACS e 20 na MEDLINE. Após a leitura dos títulos, resumos, palavras-chave, bem como os critérios de inclusão e exclusão para a pesquisa, foram excluídos 34 artigos. Sendo 26 estavam fora da temática abordada para a pesquisa; 4 por duplicidade; 2 indisponíveis para a versão completa online; 2 não disponíveis de forma gratuita.

Para elucidar a seleção dos artigos, realizou-se um fluxograma do processo de seleção dos artigos para a composição da revisão integrativa. Como demonstrado na Figura 1 a seguir:

Figura 1. Fluxograma do processo de seleção dos artigos para a revisão integrativa.

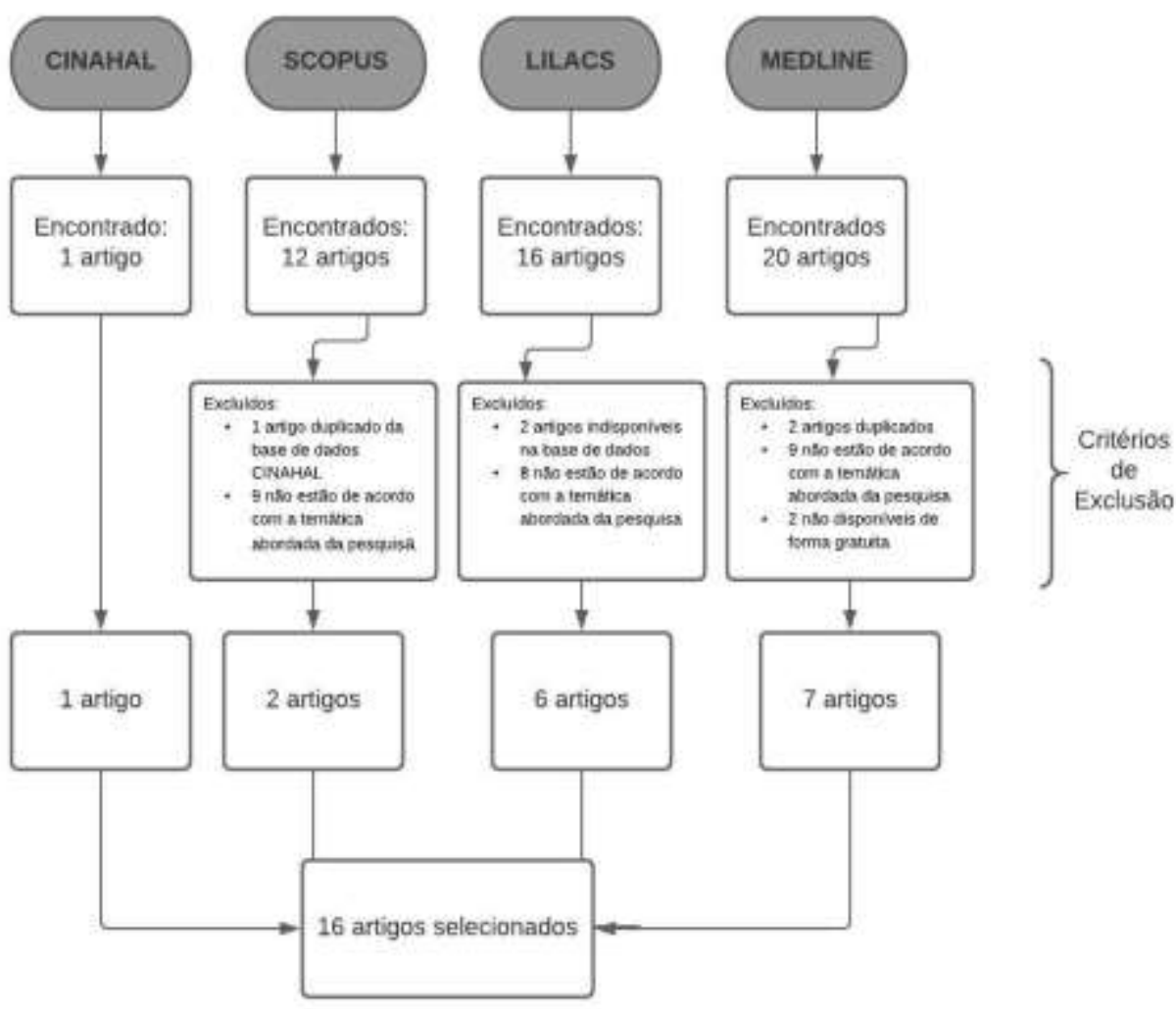

Fonte: Dados da Pesquisa (2020)

A revisão integrativa foi composta por 16 artigos, assim 1 artigo selecionado na base de dados CINAHAL, 2 na base de dados SCOPUS, 6 na LILACS por meio do portal BVS/REGIONAL, e 7 na base de dados MEDLINE. Desses artigos selecionados, foi possível constatar um significativo número nos de 2016 com 5 artigos, seguido de 2019 com 4 artigos. Contemplando também 2 artigos no ano de 2014 e 2 artigos em 2018, seguido de 1 artigo em cada ano de 2012,2013 e 2020. Os artigos foram analisados de forma crítica em relação a: número do artigo, ano, título, autores, periódico e nível de evidência, como demonstrado no Quadro 1: 
Quadro 1. Artigos selecionados para composição da revisão integrativa, de acordo com número, ano, título, autores, periódico, método e nível de evidência.

\begin{tabular}{|c|c|c|c|c|c|}
\hline $\begin{array}{l}N^{\circ} / \\
\text { Ano }\end{array}$ & Título & Autores & Periódico & Método & $\begin{array}{l}\text { Nível de } \\
\text { evidência }\end{array}$ \\
\hline $\begin{array}{c}\text { A1 } \\
2012\end{array}$ & $\begin{array}{c}\text { Let men into the pregnancy'-Men's } \\
\text { Perceptions About Being Tested for } \\
\text { Chlamydia And HIV during } \\
\text { pregnancy }\end{array}$ & $\begin{array}{l}\text { Monica Christianson, } \\
\text { Jens Boman e Birgitta } \\
\text { Essén. }\end{array}$ & Midwifery/ & Qualitativo & 4 \\
\hline $\begin{array}{c}\text { A2 } \\
2013\end{array}$ & $\begin{array}{c}\text { Men's Involvement in antenatal care } \\
\text { and labour: Rethinking a medical } \\
\text { model }\end{array}$ & $\begin{array}{l}\text { Heather Draper e } \\
\text { Jonathan Ives. }\end{array}$ & Midwifery & $\begin{array}{l}\text { Artigo de } \\
\text { reflexão }\end{array}$ & 6 \\
\hline $\begin{array}{c}\mathbf{A 3} \\
2014\end{array}$ & $\begin{array}{l}\text { What do men want from antenatal } \\
\text { screening? Findings From An } \\
\text { interview study in England }\end{array}$ & $\begin{array}{l}\text { Sandi Dheensa, Alison } \\
\text { Metcalf e, Robert } \\
\text { Williams }\end{array}$ & Midwifery/ & Qualitativo & 4 \\
\hline $\begin{array}{c}\text { A4 } \\
2014\end{array}$ & $\begin{array}{l}\text { Planejamento familiar e a saúde do } \\
\text { homem na visão das enfermeiras }\end{array}$ & $\begin{array}{c}\text { Sidneia Casarin e } \\
\text { Hedi Crecencia Heckler } \\
\text { de Siqueira }\end{array}$ & $\begin{array}{l}\text { Revista de } \\
\text { Enfermagem } \\
\text { Anna Nery }\end{array}$ & Qualitativo & 4 \\
\hline $\begin{array}{c}\text { A5 } \\
2016\end{array}$ & $\begin{array}{l}\text { Profissionais de Saúde e o (não) } \\
\text { atendimento ao Homem-Pai: Análise } \\
\text { em Representações Sociais }\end{array}$ & $\begin{array}{l}\text { Mirian B Cortez, } \\
\text { Nathália M Machado e } \\
\text { Zeidi Trindade }\end{array}$ & $\begin{array}{l}\text { Psicologia em } \\
\text { Estudo / } \\
\text { Brasil }\end{array}$ & Qualitativo & 4 \\
\hline $\begin{array}{c}\text { A6 } \\
2016\end{array}$ & $\begin{array}{l}\text { Linhas de cuidados masculinos } \\
\text { voltados para a saúde sexual, a } \\
\text { reprodução e a paternidade }\end{array}$ & $\begin{array}{l}\text { Romeu Gomes, } \\
\text { Lidianne Albernaz, } \\
\text { Cláudia Ribeiro et al. }\end{array}$ & $\begin{array}{c}\text { Ciência \& } \\
\text { Saúde Coletiva }\end{array}$ & $\begin{array}{l}\text { Trabalho } \\
\text { opinativo/ } \\
\text { experiência }\end{array}$ & 5 \\
\hline $\begin{array}{c}\text { A7 } \\
2016\end{array}$ & $\begin{array}{l}\text { E agora o homem vem?! Estratégias } \\
\text { de atenção à saúde dos homens }\end{array}$ & $\begin{array}{l}\text { Martha Cristina Nunes } \\
\text { Moreira, } \\
\text { Romeu Gomes e } \\
\text { Claudia Regina Ribeiro }\end{array}$ & $\begin{array}{l}\text { Cadernos de } \\
\text { Saúde Pública }\end{array}$ & $\begin{array}{l}\text { Quantitativo/ } \\
\text { Qualitativa }\end{array}$ & 4 \\
\hline $\begin{array}{c}\mathbf{A 8} \\
2016\end{array}$ & $\begin{array}{l}\text { Saberes e Práticas de homens perante } \\
\text { o planejamento reprodutivo }\end{array}$ & $\begin{array}{l}\text { Ana Carolina da Silva } \\
\text { Coelho, Adriana Lemos } \\
\text { Pereira e Carla Cardi } \\
\text { Nepomuceno }\end{array}$ & $\begin{array}{l}\text { Revista de } \\
\text { Enfermagem } \\
\text { do Centro } \\
\text { Oeste Mineiro }\end{array}$ & Qualitativo & 4 \\
\hline $\begin{array}{c}\text { A9 } \\
2016\end{array}$ & $\begin{array}{l}\text { Encontros e desencontros entre a } \\
\text { saúde do homem, a promoção da } \\
\text { paternidade participativa e a saúde } \\
\text { sexual e reprodutiva na atenção básica }\end{array}$ & $\begin{array}{l}\text { Cláudia Ribeiro, } \\
\text { Romeu Gomes e } \\
\text { Martha Cristina } \\
\text { Moreira }\end{array}$ & $\begin{array}{l}\text { Revista Saúde } \\
\text { \& Coletiva }\end{array}$ & $\begin{array}{l}\text { Quantitativo/ } \\
\text { Qualitativa }\end{array}$ & 4 \\
\hline $\begin{array}{l}\text { A10 } \\
2018\end{array}$ & $\begin{array}{c}\text { Participação do homem no } \\
\text { planejamento reprodutivo: revisão } \\
\text { integrativa }\end{array}$ & $\begin{array}{l}\text { Isabela Lima Nogueira, } \\
\text { Simone Mendes } \\
\text { Carvalho, Florence } \\
\text { Romijn Tocantins et al. }\end{array}$ & $\begin{array}{l}\text { Revista Online } \\
\text { de Pesquisa } \\
\text { Cuidado é } \\
\text { Fundamental }\end{array}$ & $\begin{array}{c}\text { Revisão } \\
\text { Integrativa }\end{array}$ & 4 \\
\hline $\begin{array}{c}\text { A11 } \\
2018\end{array}$ & $\begin{array}{c}\text { Maternal Health: a Qualitative Study } \\
\text { of Male Partners' Participation in } \\
\text { Lagos, Nigeria }\end{array}$ & $\begin{array}{l}\text { Samuel Adejoh, } \\
\text { Adetayo Olorunlana e } \\
\text { Olatunji Olaosebikan }\end{array}$ & $\begin{array}{l}\text { International } \\
\text { Journal of } \\
\text { Behavioral } \\
\text { Medicine }\end{array}$ & Qualitativo & 4 \\
\hline $\begin{array}{l}\text { A12 } \\
2019\end{array}$ & $\begin{array}{l}\text { Participação paterna no ciclo } \\
\text { gravídico-puerperal e seus efeitos } \\
\text { sobre a saúde da mulher }\end{array}$ & $\begin{array}{l}\text { Thais Rafaela Lira } \\
\text { Cavalcanti e Viviane } \\
\text { Rolim de Hollanda }\end{array}$ & $\begin{array}{l}\text { Revista } \\
\text { Enfermagem } \\
\text { m Foco }\end{array}$ & $\begin{array}{l}\text { Revisão } \\
\text { Integrativa }\end{array}$ & 4 \\
\hline
\end{tabular}




\begin{tabular}{|c|c|c|c|c|c|}
\hline $\begin{array}{l}\mathbf{A 1 3} \\
2019\end{array}$ & $\begin{array}{l}\text { The effect of paternal cues in prenatal } \\
\text { care settings on men's } \\
\text { involvement intentions }\end{array}$ & $\begin{array}{l}\text { Analia F. Albuja, Diana } \\
\text { T. } \\
\text { Sanchez, Shawna J. Lee } \\
\text { et al. }\end{array}$ & PLOS ONE & $\begin{array}{l}\text { Estudo } \\
\text { controlado }\end{array}$ & 1 \\
\hline $\begin{array}{l}\mathbf{A 1 4} \\
2019\end{array}$ & $\begin{array}{c}\text { Barriers To Women's Participation in } \\
\text { perinatal care: a qualitative study in } \\
\text { Iran }\end{array}$ & $\begin{array}{l}\text { Vahideh Firouzan, } \\
\text { Mahnaz Noroozi, Ziba } \\
\text { Farajzadeganand et al. }\end{array}$ & $\begin{array}{l}\text { BMC } \\
\text { Pregnancy and } \\
\text { Childbirth }\end{array}$ & Qualitativo & 4 \\
\hline $\begin{array}{l}\text { A15 } \\
2019\end{array}$ & $\begin{array}{l}\text { Preparation for fatherhood: A survey } \\
\text { of men's preconception health } \\
\text { knowledge and behaviour in England }\end{array}$ & $\begin{array}{l}\text { Jill Shawe, Dilisha } \\
\text { Patel, Mark Joy et al }\end{array}$ & PLOS ONE & Quantitativo & 4 \\
\hline $\begin{array}{l}\text { A16 } \\
2020\end{array}$ & $\begin{array}{l}\text { PRENACEL partner - use of short } \\
\text { message service (SMS) to encourage } \\
\text { male involvement in prenatal care: a } \\
\text { cluster randomized trial }\end{array}$ & $\begin{array}{l}\text { Lívia Bonifácio, Ana } \\
\text { Carolina Franzon, } \\
\text { Fabiani Zaratini et al. }\end{array}$ & $\begin{array}{l}\text { Reproductive } \\
\text { Health }\end{array}$ & $\begin{array}{c}\text { Estudo } \\
\text { Controlado }\end{array}$ & 1 \\
\hline
\end{tabular}

Fonte: Dados da pesquisa (2020).

Ao que se refere à procedência geográfica, 9 estudos foram desenvolvidos no Brasil (A4, A5, A6, A7, A8, A9, A10, A12, A16), seguido da Inglaterra com 3 estudos (A2, A3, A15). Houve representatividade de 1 estudo em cada um dos respectivos países: Irã (A14), Nigéria (A11), Estados Unidos (A13) e Suécia (A1).

Referente a metodologia dos artigos selecionados, houve predominância de estudos de abordagem qualitativa em 7 estudos (A1, A3, A4, A5, A8, A11, A14), seguido de 2 estudos com abordagem quantitativa/qualitativa (A7, A9) e de 2 estudos controlados (A13, A16). Artigos de revisão integrativa apresentaram-se em 2 estudos (A10, A12), e por fim, 1 estudo de reflexão (A2), 1 estudo como forma de trabalho opinativo/reflexivo (A6) e 1 estudo com abordagem quantitativa (A15).

O nível de evidência da maioria dos artigos foi considerado baixo. Em sua maioria, apresentou-se estudos em Nível de evidência 4 (A1, A3, A4, A5, A7, A8, A9, A10, A11, A12, A14, A15), Nível de evidência 6 com 1 estudo (A2), Nível evidência 5 (A6) e Nível de evidência 1 com 2 estudos (A13, A16).

Ao realizar a análise dos periódicos encontrados, observa-se que o quantitativo de periódicos nacionais e internacionais são similares. Representado por 8 internacionais (A1, A2, A3, A11, A13, A14, A15, A16) e 8 nacionais (A4, A5, A6, A7, A8, A19, A10, A12).

O universo amostral foi composto em maioria por 8 publicações pertencentes à área de saúde coletiva (A4, A6, A7, A8, A9, A10, A12, A16). Seguida de 7 estudos da área de enfermagem (A1, A2, A3, A4, A8, A10, A12), destas 3 são internacionais (A1, A2, A3), a especificação do trabalho das Midwives, 1 estudo de psicologia (A5).

Após realizada a caracterização e análise dos artigos selecionados, foram formadas três categorias para a discussão e apresentação desta revisão integrativa: 1) Planejamento reprodutivo e o foco na prevenção e rastreio de infecções sexualmente transmissíveis, 2) O homem-pai como coadjuvante nas ações de saúde e a atenção ao binômio mãe-bebê, 3) Dificuldades e estratégias de inclusão aos pais no período pré-natal pelos serviços e profissionais de saúde.

\section{Discussão}

\section{Planejamento reprodutivo e foco na prevenção e rastreio de infecções sexualmente transmissíveis.}

Ao referir as ações de cuidado durante o período pré-natal, destaca-se que estes são centrados majoritariamente no serviço de atenção primária à saúde, ou seja, nas unidades básicas de saúde. Dessa forma, pretende-se aumentar a presença masculina nesse nível de atenção, o qual é a porta de entrada preferencial no Sistema Único de Saúde, além de ser responsável pela promoção à saúde e prevenção de doenças, como está no cerne da Política Nacional de Atenção Integral à Saúde do Homem 
(Brasil, 2008). Outro ponto importante para destacar, é a crescente disseminação do SUS na estratégia do Pré-Natal do Parceiro, que visa, por um lado colaborar para o exercício da paternidade e cuidado e, por outro servir como porta de entrada positiva para os homens, integrando-os na lógica dos serviços de saúde, ofertados, possibilitando que realizem por exemplo, exames preventivos, tais como anti HIV, Sífilis e hepatites, hipertensão, diabetes e atualizem sua carteira de vacinação, entre outros. (Reis \& Pereira, 2017). Logo, é cabível que se tenha um número significativo de estudos ambientados na atenção básica (A4, A5, A6, A7, A8, A10, A16).

A atenção básica é de responsabilidade dos municípios e deve ser o espaço de desenvolvimento das políticas e programas ministeriais, dentre elas a PNAISH, que deve ser articulada às demais políticas específicas para outras populações (Brasil, 2008). No entanto, não é isso que tem ocorrido (A9). Os estudos demonstraram que a presença dos homens nos serviços de atenção primária ainda se mostra incipiente e as políticas, desarticuladas (A4, A5, A6, A7, A8, A9) Porém, foi percebido que os homens procuram mais os serviços de saúde quando estão envolvidos especificamente nas ações de planejamento reprodutivo (A8, A9, A10).

A maior presença dos homens no serviço de saúde, no que diz respeito ao planejamento reprodutivo, está relacionada à busca e obtenção de métodos contraceptivos, em especial, os preservativos. Também é notável a procura destes homens para a realização de cirurgias de esterilização, tais como vasectomias e a laqueadura (A4, A8, A9, A10). No entanto é necessário que o homem e a mulher que desejem realizar tais cirurgias, realizem ações educativas de planejamento reprodutivo com intuito de conseguir realizar tanto a vasectomia quanto a laqueadura.

A participação do homem no planejamento reprodutivo é um direito instituído na Lei n 9.263 , de 12 de janeiro de 1996 (Brasil, 1996). Isto quer dizer que os homens também têm todo o direito a participar da escolha e do melhor momento para ser pai. Contudo, grande parcela dos brasileiros, ainda desconhece seus direitos de como compartilhar o período gestacional, do trabalho de parto e puerpério de suas companheiras. (Reis \& Pereira, 2017).

Enfatiza-se um estudo (A9) que aponta que na região sudeste do Brasil, a vasectomia é o principal motivo para os homens procurarem o serviço de atenção primária. Entretanto, o mesmo estudo constatou que não há articulação das ações de saúde voltadas ao planejamento reprodutivo com a promoção da paternidade. $\mathrm{O}$ que deixa a desejar quanto à promoção da integralidade do cuidado masculino: quando existe um programa de planejamento reprodutivo, não existe um programa em que o homem-pai seja colocado como sujeito do cuidado e de direito à sua saúde. E quando existe um programa em que o homempai é assistido em suas necessidades básicas de saúde durante o pré-natal, não existe um programa de planejamento reprodutivo.

Os estudos também apontaram que as ações de planejamento reprodutivo ainda são direcionadas ao público feminino. Tornando-se uma atribuição feminina o dever e a preocupação sobre o planejamento reprodutivo, isentando os homens de tais preocupações. Percebe-se um desdobramento com relação ao gênero e à masculinidade que também pode ser apontado como entrave à inclusão dos homens no planejamento reprodutivo (A1, A2, A3, A4, A8, A14, A16).

Ao que se refere ao planejamento reprodutivo, que anteriormente era denominado como planejamento familiar, a Constituição Federal Brasileira de 1988 (Brasil, 1988) em seu capítulo VII - da Família, da Criança do Adolescente, do Jovem e do Idoso - afirma que este é de livre-arbítrio do casal, devendo o Estado disponibilizar recursos educacionais e científicos para garantir o exercício desse direito, enquanto um dos princípios da dignidade da pessoa humana e da paternidade responsável.

Outro motivo que leva os homens às unidades de atenção básica é a realização de testes rápidos para detecção de infecções sexualmente transmissíveis (IST), tais como HIV, sífilis e hepatites (A4, A8, A10). As IST são um grande agravo para a saúde pública, pois são responsáveis pela diminuição na qualidade de vida das pessoas, nas relações pessoais, familiares e sociais (Brasil, 2015). Segundo a Organização Mundial da Saúde, mais de um milhão de pessoas se contaminam diariamente com uma IST (Brasil, 2015). A vulnerabilidade às IST está intimamente relacionada à exposição ao risco em decorrência da falta, principalmente de conhecimentos, atitudes e práticas favoráveis à saúde sexual (Santos et al.,2018). 
Foi apontado que o pouco conhecimento que os homens tinham a respeito do planejamento reprodutivo, além da obtenção de métodos contraceptivos, estava relacionado com a preocupação de transmitir doenças e/ou se contaminar durante as atividades sexuais (A8).

Em um estudo internacional (A15) foi observado que os homens que participaram do planejamento reprodutivo tiveram ao menos uma mudança positiva em seu comportamento, relacionada à sua saúde. É importante destacar que metade dos homens do estudo tinha excesso de peso e um terço estava sob medicação que poderia prejudicar a reprodução, além de uma alta prevalência no consumo de álcool e fumo. O que indica uma necessidade de uma maior conscientização para os homens no cuidado à sua saúde, principalmente no período pré-concepcional, ou seja, no planejamento reprodutivo. Enfatizando que a procura destes homens nas ações de planejamento reprodutivo pode também ser oportuna para as ações de cuidado à saúde dos homens.

\section{O homem-pai como coadjuvante nas ações de saúde e a atenção ao binômio mãe-bebê}

Os estudos abordaram de maneira enfática o papel do homem-pai como coadjuvante do processo de cuidado à gestação, parto, pós-parto e puerpério. Dando ênfase às ações de cuidado ao binômio mãe-bebê (A9, A10, A11, A12, A13).

No estudo que investigou as ações de promoção à saúde do homem articuladas ao -pré-natal masculino -e - pré-natal do parceiro - percebeu-se que, apesar de serem nomeados com as palavras "parceiro" e "masculino", os cuidados estão voltados para o binômio mãe-bebê, enquanto o homem-pai ocupa o papel de auxiliar na promoção da saúde do referido par e de coadjuvante em uma ação que leva o seu nome (A5).

Neste mesmo estudo, ao que se refere à região sul do Brasil, mostra uma resistência dos profissionais de saúde para direcionar as ações de cuidado aos homens. As diferenças no tratamento ofertado aos homens por médicos(as) e enfermeiros (as) nas consultas de pré-natal, sendo os(as) médicos(as) os(as) mais resistentes à inclusão dos homens no pré-natal, parto e pós-parto (A5). Em outro estudo, notou-se que a figura do homem-pai era apresentada de forma idealizada por médicos e enfermeiros e referida como importante, embora os homens ainda sejam considerados coadjuvantes por estes profissionais nos serviços de atenção reprodutiva (A12).

Sousa et al (2021) corrobora ao constatar que há pouca habilidade dos profissionais que realizam assistência pré-natal diante da inclusão e manejo do cuidado dos homens-pais durante as consultas. O que pode acarretar nas ausências dos homenspais as consultas e a não vinculação ao serviço de saúde.

Um estudo ao qual foi realizada uma revisão integrativa (A12) reuniu trabalhos que abordaram o envolvimento do homem-pai desde o início do período gestacional ao buscarem desenvolver melhores atitudes diante da complexidade e particularidades da gravidez, parto e puerpério. Ressaltou-se nas buscas desta revisão as repercussões para a saúde do binômio mãe-bebê, mas não foram evidenciadas repercussões na saúde do parceiro. Esse resultado confirma a pouca preocupação na promoção da saúde dos homens, assim como nas ações voltadas para o estímulo à paternidade.

O binômio mãe-bebê são considerados as partes frágeis e vulneráveis do conjunto, contrastando em oposição aos homens. Com essas percepções, permeadas pela cultura de gênero, que vêm impedindo que os profisssionais de saúde e até mesmo os próprios homens, de também se perceberem como sujeitos de cuidado e direito à saúde. Levando assim, a ações voltadas para a paternidade que não conseguem incluir verdadeiramente os homens-pais nas ações de cuidado em geral, e de se articularem com ações que historicamente têm as mulheres e crianças como público alvo (Ribeiro et al., 2017).

\section{Dificuldades e estratégias de inclusão aos homens-pais no período pré-natal pelos serviços e profissionais de saúde}

Os estudos apontam quanto as dificuldades na inserção dos homens-pais ao serviço de saúde e em especial ao pré-natal, está relacionada ao papel destes em de provedor familiar e o receio de se ausentar das práticas laborais (A4, A8, A9, A10, A14). 
O principal empecilho para a presença dos homens nos serviços de saúde é o horário de funcionamento, majoritariamente diurno. Outra queixa é a longa espera para atendimento e pouca resolutividade do serviço, fazendo com que tenham que voltar outras vezes. Dessa forma, optar pelos serviços de emergência e pronto atendimento têm sido historicamente uma estratégia dos homens quando acometidos por algum problema de saúde.

Essas designações atravessam e organizam as práticas da assistência, contribuindo na construção da ideia de uma restituição rápida da força física masculina, a sua força de trabalho. Assim, é mais uma medida de articulação que leva os homens a procurarem mais os serviços de emergência e os hospitais, do que de fato o serviço de atenção primária, em práticas de promoção à saúde (Schraiber \& Figueiredo, 2011).

O ambiente do serviço de saúde também se mostra como barreira em relação à presença dos homens-pais (A4, A5, A6, A7, A8, A10, A11, A16). A percepção de um espaço feminilizado, composto, basicamente, por profissionais mulheres e frequentado por uma clientela essencialmente feminina, também é apontado nos artigos como um entrave aos homens. Tal situação provocaria nos homens a sensação de não pertencimento àquele espaço (A4) A literatura evidencia que a histórica ausência masculina nos serviços de saúde tem em sua raiz o entendimento do que é ser homem e aspectos relacionados à masculinidade (Gomes, 2010; Vieira et al.,2013; Silva et al.,2012).

Uma questão importante levantada nos artigos, se refere à qualificação profissional dos profissionais pré-natalistas, médicos e enfermeiros que, em sua boa parte, não incentivam a presença paterna nos atendimentos às gestantes e/ou os ignoram nas consultas, não se dirigindo a eles durantes as conversas e orientações (A5, A6, A7, A9).

Um estudo também apontou lacunas e deficiências na formação acadêmica, o que também impacta negativamente a consolidação dos preceitos da PNAISH. Tem-se a necessidade de enfrentar essas lacunas com maior naturalidade, levar o tema da saúde do homem e da paternidade às mídias e à formação dos profissionais de saúde (A7).

Para Lima \& Barbosa (2020) é relevante considerar que os serviços de saúde estão relacionados às dificuldades de inclusão destes homens-pais no pré-natal. O profissional enfermeiro como integrante da equipe de saúde, e sendo um dos profissionais pré-natalistas na rede de atenção básica, têm como atribuição acolher este pai. Principalmente incluí-lo nas consultas para que este se expresse, retire suas dúvidas, expectativas, além de estar junto as práticas de cuidado a sua saúde e do binômio.

O uso de tecnologias de comunicação também foi uma estratégia de inclusão dos homens-pais aos serviços de saúde e ao pré-natal. Este esteve presente em estudo brasileiro, onde o cenário foi uma unidade básica de saúde de Ribeirão Preto - São Paulo (A16). O estudo traz como estratégia de educação em saúde, a utilização de tecnologias de comunicação, ao qual parece ser uma ferramenta útil para o cuidado pré-natal. Esta teve boa aceitabilidade e uma grande inclusão no acesso de homens-pais aos serviços de pré-natal, parto e pós-parto. Neste referido estudo, a tecnologia de comunicação utilizada foi o PRENACEL, em que se os homens-pais recebiam periodicamente em seus celulares mensagens de textos via SMS. As mensagens incluíam informações a respeito da gestação, parto e pós parto.

No contexto internacional, estudos que objetivam traçar estratégias para uma maior inclusão dos homens-pais nos serviços de saúde e analisar os fatores que impedem o envolvimento destes no período pré-natal, logo, é necessário uma efetivação de ações de saúde e promoção para a paternidade (A1, A2, A3, A13, A15).

O estudo que foi desenvolvido por Christianson, Boman e Essén (2012) enfatizou a importância dos exames de sorologia HIV e clamídia aos dos homens-pais, como uma forma de melhorar os cuidados à saúde desta população, e sobretudo incluir nos cuidados ao binômio mãe-bebê. Dessa maneira, a oferta de testes sorológicos aos homens-pais no primeiro trimestre de gravidez é uma estratégia para melhorar a saúde dos homens, de suas parceiras e de seus bebês, sendo uma oportunidade para as práticas de inclusão ao homem-pai à sua saúde (A1). 
Destaca-se o papel das midwives, ao qual se colocam na posição de educar homens-pais, e ressaltam a importância de mudar o papel dos homens em relação a ser o sujeito do cuidado. As midwives são consideradas como motivadoras significativas destes testes para os homens-pais (A1).

Constata-se na literatura nacional que as enfermeiras obstétricas compreendem o papel do homem no acompanhamento do pré-natal como uma forma de se prepararem para o parto. Enfatizando um cuidado a este homem de acolhimento, com orientações necessárias, em linguagem clara e acessível. Deve ser oferecida também uma escuta ativa a estes homens, principalmente nos grupos educativos, refletindo e disseminando conhecimentos para evitar a redução do seu papel a um simples coadjuvante nas atividades relativas ao pré-natal (Balica \& Aguiar, 2019).

Percebe-se que as estratégias quanto ao contexto dos estudos internacionais vão além de práticas de saúde concretas em si. O Royal College of Midwives (2011), estabeleceu em suas diretrizes, o envolvimento de pais, incluindo a utilização dos seus nomes, e espaços para que estes expressem seus pensamentos e sentimentos. De forma que se sintam bem vindos e envolvidos nas práticas de cuidado com as midwives.

A medicalização do cuidar também apareceu entre os estudos. Destaca-se que há uma preocupação ao que se refere quanto aos cuidados aos homens no período pré-natal. Para que estas práticas não se tornem medicalizadas, assim como as do binômio mãebebê. Em que podem ser observadas somente com a prescrição de exames, rastreio de infecções e ações de saúde sem ao menos conhecer as necessidades dos homens-pais (A3).

Firouzan, et al (2019) em seu estudo situado na Nigéria (A11), relata que uma das barreiras para a inclusão do homempai no pré-natal estaria relacionada às restrições financeiras destes homens, além das exigências do trabalho e o tempo que são disponibilizados para as consultas de pré-natal. Isto foi evidenciado ainda mais entre homens-pais artesãos e comerciantes, pois os mesmos alegaram não possuir rendimentos fixos, sendo autônomos em seu trabalho. Foi descrito neste estudo, o depoimento de um homem, que alega não poder estar presente às consultas, pois não sabe como irá sustentar a família, no caso de se ausentar de suas atividades laborais.

No Irã, em um estudo (A14) também detalhou que a principal barreira para a inclusão dos homens nas ações de saúde do pré-natal está relacionada aos aspectos culturais, pessoais, socioeconômicos e principalmente ao sistema de saúde local. De acordo com os homens-pais participantes desta pesquisa, a falta de tempo e a exaustão causada pelas longas horas de trabalho destes homens, associada a falta de plano de licença para os pais na maioria das organizações e institutos de trabalho são uma barreira para a participação destes, principalmente ao que se refere ao envolvimento na gravidez, parto e cuidados pós-parto. Além disso, demonstraram-se a falta de apoio dos empregadores para os homens que gostariam de usar esta licença, mas também ficou evidenciado o medo dos homens de perderem seus empregos ao usarem a licença que seria concedida.

A literatura brasileira aponta que um dos impeditivos para a presença dos homens nas consultas de pré-natal seria o papel dos homens de provedores da família. Além do fato do medo de perderem seus empregos e fragilizam suas relações de trabalho. O tempo de espera, e o horário de funcionamento das unidades básicas de saúde pode fazer com que estes percam seu dia de trabalho e não tenham liberação por parte de sua chefia. (Ribeiro et al., 2017).

\section{Conclusão}

Foi constatado nos estudos a pouca presença do homem nos serviços de saúde, mas quando estes estão no serviço, são majoritariamente envolvidos nas ações de planejamento reprodutivo. A presença destes homens está associada à obtenção de métodos contraceptivos como preservativos, mas também para métodos contraceptivos cirúrgicos, como vasectomia e laqueadura no caso das mulheres. 
A linha de cuidado a este homem-pai é inexistente, ou seja, este homem somente participa das ações de planejamento reprodutivo, mas não tem uma continuidade na integralidade de sua saúde. Notando-se uma não articulação entre ações de planejamento reprodutivo e pré-natal do parceiro e vice e versa.

O homem ainda é coadjuvante nas práticas de cuidado ao que se refere aos cuidados no período pré-natal, este ainda muito direcionado à saúde no binômio mãe-bebê. O que também está relacionado a assistências dos profissionais que realizam as consultas de pré-natal. Estes profissionais possuem pouca habilidade quanto às práticas de cuidado aos homens-pais. Sendo julgados pelos próprios profissionais que é importante que o homem esteja no serviço de saúde, mas estes não realizam cuidados voltados para os homens-pais.

Ressalta-se que nenhum estudo brasileiro e internacional evidenciaram de fato ações direcionadas ao pré-natal do parceiro, ou a realização deste programa nestes espaços. Encontraram-se estudos aos quais abordam as dificuldades e estratégias de ação para o acesso aos homens durante o período pré-natal. Portando, sugerimos que mais estudos sejam realizados a respeito da temática, visto que ainda se encontram grande parte dos estudos de forma qualitativa e ensaios teóricos. É necessário que mais produções investiguem a respeito da temática para melhor consolidação das Políticas Públicas e Programas relacionadas à saúde de homens-pais e seu papel no ciclo gravídico-puerperal.

\section{Referências}

Adejoh, S. O., Olorunlana, A., \& Olaosebikan, O. (2018). Maternal health: A qualitative study of male partners' participation in Lagos, Nigeria. International journal of behavioral medicine, 25(1), 112-122. https://www.researchgate.net/publication/317387051_Maternal_Health _a_Qualitative_Study_of_Male_Partners'_Participation_in_Lagos_Nigeria

Albuja, A. F., Sanchez, D. T., Lee, S. J., Lee, J. Y., \& Yadava, S. (2019). The effect of paternal cues in prenatal care settings on men's involvement intentions. PloS one, 14(5), 1-15. https://journals.plos.org/plosone/article?id=10.1371/journal.pone.0216454

Balica, L. O., \& Aguiar, R. S. (2019). Percepções paternas no acompanhamento do pré-natal. Revista de Atenção à Saúde, 17(61), 114-126. https://seer.uscs.edu.br/index.php/revista_ciencias_saude/article/view/5934

Branco, V. M. C., \& Carvalho, M. L. M. (2012, junho). Unidades de Saúde Parceiras do Pai: uma estratégia para favorecer a inclusão dos homens nos serviços de saúde. In $10^{\circ}$ Congresso Internacional da Rede Unida. Rio de Janeiro, RJ. http://primeirainfancia.org.br/wp-content/uploads/2016/04/unidade-de-sac3badeparceira-do-pai.pdf

Brasil. (2015). Ministério da Saúde. Protocolo Clínico e Diretrizes Terapêuticas para Atenção Integral às Pessoas com Infecções Sexualmente Transmissíveis Ministério da Saúde. Brasília: Ministério da Saúde

Brasil. (2008). Ministério da Saúde. Política Nacional de Atenção Integral à Saúde do Homem. Ministério da Saúde. Ministério da Saúde

Bonifácio, L. P., Franzon, A. C. A., Zaratini, F. S., Vicentine, F. B., Barbosa-Júnior, F., Braga, G. C., \& Vieira, E. M. (2020). PRENACEL partner-use of short message service (SMS) to encourage male involvement in prenatal care: a cluster randomized trial. Reproductive health, 17(1), 1-12. https://www.ncbi.nlm.nih.gov/pmc/articles/PMC7132868/

Casarin, S. T., \& Siqueira, H. C. H. D. (2014). Planejamento familiar e a saúde do homem na visão das enfermeiras. Escola Anna Nery, 18(4), 662-668. https://www.scielo.br/pdf/ean/v18n4/1414-8145-ean-18-04-0662.pdf

Cavalcanti, T. R. L., \& Holanda, V. R. D. (2019). Participação paterna no ciclo gravídico-puerperal e seus efeitos sob a saúde da mulher. Enferm. foco (Brasília), 93-98. http://revista.cofen.gov.br/index.php/enfermagem/article/view/1446

Christianson, M., Boman, J., \& Essén, B. (2013). 'Let men into the pregnancy'-Men's perceptions about being tested for Chlamydia and HIV during pregnancy. Midwifery, 29(4), 351-358. https://pubmed.ncbi.nlm.nih.gov/22417755/

Cortez, M. B., Machado, N. M., Trindade, Z. A., \& Souza, L. G. S. (2016). Profissionais de saúde e o (não) atendimento ao homem-pai: análise em representações sociais. Psicologia em Estudo, 21(1), 53-63. https://periodicos.uem.br/ojs/index.php/PsicolEstud/article/view/28323

da Silva Coelho, A. C., Pereira, A. L., \& Nepomuceno, C. C. (2016). Saberes e práticas de homens perante o planejamento reprodutivo. Revista de Enfermagem do Centro-Oeste Mineiro, 6(3), 2398-2409. http://www.seer.ufsj.edu.br/index.php/recom/article/view/1079

de Sousa, S. C., de Oliveira, F. B. M., Sousa, F. D. C. A., Silva, S. S., da Silva, W. C., Lima, K. L. A., \& da Silva, R. A. (2021). Assistência ao pré-natal: participação do pai na gestação saudável. Research, Society and Development, 10(1), e14710111330-e14710111330. https://rsdjournal.org/index.php/rsd/article/view/11330/10345

Dalfovo, M. S., Lana, R. A., \& Silveira, A. (2008). Métodos quantitativos e qualitativos: um resgate teórico. Revista interdisciplinar científica aplicada, 2(3), 1-13. https://rica.unibes.com.br/rica/issue/view/18 
Dheensa, S., Metcalfe, A., \& Williams, R. (2015). What do men want from antenatal screening? Findings from an interview study in England. Midwifery, 31(1), 208-214. https://www.sciencedirect.com/science/article/abs/pii/S0266613814002216

Draper, H., \& Ives, J. (2013). Men's involvement in antenatal care and labour: Rethinking a medical model. Midwifery, 29(7), 723-729. https://pubmed.ncbi.nlm.nih.gov/23522667/

Federal, S. (1998). Constituição da República Federativa do Brasil, 1988. http://www.planalto.gov.br/ccivil_03/constituicao/constituicao.htm

Firouzan, V., Noroozi, M., Farajzadegan, Z., \& Mirghafourvand, M. (2019). Barriers to men's participation in perinatal care: a qualitative study in Iran. BMC pregnancy and childbirth, 19(1), 1-9. https://bmcpregnancychildbirth.biomedcentral.com/articles/10.1186/s12884-019-2201-2

Gomes, R. (2003). Sexualidade masculina e saúde do homem: proposta para uma discussão. Ciência \& Saúde Coletiva, 8(3), 825-829. https://www.scielo.br/scielo.php?script=sci_arttext\&pid=S1413-81232003000300017

Gomes, R., Albernaz, L., Ribeiro, C. R. S., Moreira, M. C. N., \& Nascimento, M. (2016). Linhas de cuidados masculinos voltados para a saúde sexual, a reprodução e a paternidade. Ciência \& Saúde Coletiva, 21, 1545-1552. https://www.scielo.br/scielo.php?pid=S1413$81232016000501545 \&$ script=sci_abstract\&tlng=pt

Gomes, R., Nascimento, E. F. D., \& Araújo, F. C. D. (2007). Por que os homens buscam menos os serviços de saúde do que as mulheres? As explicações de homens com baixa escolaridade e homens com ensino superior. Cadernos de Saúde Pública, 23, 565-574. https://www.scielo.br/scielo.php?pid=S0102311X2007000300015\&script=sci_abstract\&tlng=pt

Herrmann, A. (2016). Guia de Saúde do Homem para Agente Comunitário de Saúde (ACS). Ministério da saúde, Rio de Janeiro, RJ. https://central3.to.gov.br/arquivo/369121/

Kabagenyi, A., Jennings, L., Reid, A., Nalwadda, G., Ntozi, J., \& Atuyambe, L. (2014). Barriers to male involvement in contraceptive uptake and reproductive health services: a qualitative study of men and women's perceptions in two rural districts in Uganda. Reproductive health, 11(1), 1-9. https://pubmed.ncbi.nlm.nih.gov/24597502/

Lei n. 11.108 de 7 de abril de 2005. Altera a lei 8.080 de 19 de setembro de 1990, para garantir às parturientes o direito à presença do acompanhante durante o trabalho de parto, parto e pós-parto imediato no Sistema Único de SAÚDE - SUS. Diário Oficial da União, Brasília - DF. http://www.planalto.gov.br/ccivil_03/_Ato2004-2006/2005/Lei/L11108.htm

Lei n. 9.263 de 12 de janeiro de 1996 (BR). Regula o $§ 7^{\circ}$ do art. 226 da Constituição Federal, que trata do planejamento familiar, estabelece penalidades e dá outras providências. Diário Oficial da União, Brasília, DF. http://www.planalto.gov.br/ccivil_03/leis/L9263.htm

Lima, J. R., da Costa, L. D., \& Barbosa, S. (2020). O envolvimento paterno no acompanhamento ao pré-natal: desafios e implicações. Research, Society and Development, 9(11), e73491110559-e73491110559. https://rsdjournal.org/index.php/rsd/article/view/10559/9293

Mendes, K. D. S., Silveira, R. C. C. P., \& Galvão, C. M. (2008) Integrative literature review: a research method to incorporate evidence in health care and nursing. Texto Contexto Enferm [Internet], 17(4), 758-64. https://www.scielo.br/scielo.php?pid=S0104-07072008000400018\&script=sci_abstract

Moreira, M. C. N., Gomes, R., \& Ribeiro, C. R. (2016). E agora o homem vem?! Estratégias de atenção à saúde dos homens. Cadernos de Saúde Pública, 32 , 1-10. Recuperado em 15 de agosto, 2020, de https://www.scielo.br/scielo.php?pid=s0102-311x2016000400710\&script=sci_abstract\&tlng=pt

Nascimento, A. O. D., Marcelino, P. H. R., Vieira, R. D. S., \& Lemos, A. (2019). A importância do acompanhamento paterno no pós-parto e o exercício da paternidade. Rev. pesqui. cuid. fundam. (Online), 475-480. https://pesquisa.bvsalud.org/portal/resource/pt/biblio-969982

Nogueira, I. L., Carvalho, S. M., Tocantins, F. R., \& Freire, M. A. M. (2018). Participação do homem no planejamento reprodutivo: revisão integrativa. Rev. Pesqui.(Univ. Fed. Estado Rio J., Online), 242-247. https://pesquisa.bvsalud.org/portal/resource/pt/biblio-908414

Portaria n. 1.459 de 24 de junho de 2011. Institui, no âmbito do Sistema Único de Saúde - SUS - a Rede Cegonha. Diário Oficial da União, Brasília, DF. https://bvsms.saude.gov.br/bvs/saudelegis/gm/2011/prt1459_24_06_2011.html

Reis, A., Pereira, A. (2017). Saúde de homens: conceitos e práticas de cuidados. Águia Dourada.

Ribeiro, C. R., Gomes, R., \& Moreira, M. C. N. (2017). Encontros e desencontros entre a saúde do homem, a promoção da paternidade participativa e a saúde sexual e reprodutiva na atenção básica. Physis: Revista de Saúde Coletiva, 27, 41-60. https://www.scielo.br/scielo.php?pid=S0103$73312017000100041 \&$ script=sci_abstract\&tlng=pt

Santos, C. M. D. C., Pimenta, C. A. D. M., \& Nobre, M. R. C. (2007). The PICO strategy for the research question construction and evidence search. Revista latino-americana de enfermagem, 15(3), 508-511. https://www.scielo.br/scielo.php?pid=S0104-11692007000300023\&script=sci_abstract

Santos, C. M. A., Oliveira, J. D. S., Lima, S. V. M. A., Santos, A. D. D., Góes, M. A. D. O., \& Sousa, L. B. D. (2018). Conhecimentos, atitudes e prática de homens sobre infecções sexualmente transmissíveis. Cogitare Enfermagem, 23(1), 1-8. https://revistas.ufpr.br/cogitare/article/view/54101

Schraiber, L. B., \& Figueiredo, W. S. (2011). Integralidade em saúde e os homens na perspectiva relacional de gênero. In: R. Gomes, Saúde do Homem em debate (Cap x, pp. 19-38). Fiocruz. http://books.scielo.org/id/6jhfr/pdf/gomes-9788575413647-02.pdf

Shawe, J., Patel, D., Joy, M., Howden, B., Barrett, G., \& Stephenson, J. (2019). Preparation for fatherhood: a survey of men's preconception health knowledge and behaviour in England. PLoS One, 14(3), 1-11. https://journals.plos.org/plosone/article?id=10.1371/journal.pone.0213897

Silva, P. A. D. S., Furtado, M. D. S., Guilhon, A. B., Souza, N. V. D. D. O., \& David, H. M. S. L. (2012). A saúde do homem na visão dos enfermeiros de uma unidade básica de saúde. Escola Anna Nery, 16(3), 561-568. https://www.scielo.br/scielo.php?pid=S1414-81452012000300019\&script=sci_abstract\&tlng=pt 
Research, Society and Development, v. 10, n. 4, e59310414460, 2021

(CC BY 4.0) | ISSN 2525-3409 | DOI: http://dx.doi.org/10.33448/rsd-v10i4.14460

Sousa, L. M. M., Marques, J. M., Firmino, C. F., Frade, F., Valentim, O. S., \& Antunes, A. V. (2018). Frameworks to research question in evidence-based practice. Rev Investig.Enferm [Internet], 2(23), 31-39. https://repositorio-cientifico.essatla.pt/handle/20.500.12253/1287

Souza, M. T., Silva, M. D., \& Carvalho, R. (2010). Integrative review: what is it? How to do it? Einstein [Internet], 8(1), 102-106. http://www.scielo.br/pdf/eins/v8n1/pt_1679-4508-eins-8-1-0102.pdf

Stetler, C. B., Morsi, D., Rucki, S., Broughton, S., Corrigan, B., Fitzgerald, J., \& Sheridan, E. A. (1998). Utilization-focused integrative reviews in a nursing service. Applied Nursing Research, 11(4), 195-206. https://pubmed.ncbi.nlm.nih.gov/9852663/

Vieira, K. L. D., de Oliveira, V. L. G., Borba, M. R., \& da Silva, C. F. C. (2013). Atendimento da população masculina em unidade básica saúde da família: motivos para a (não) procura. Escola Anna Nery Revista de Enfermagem, 17(1), 120-127. https://www.scielo.br/scielo.php?pid=S1414$81452013000100017 \&$ script=sci_abstract\&tlng=pt

Whittemore, R., \& Knafl, K. (2005). The integrative review: updated methodology. Journal of advanced nursing, 52(5), 546-553. https://pubmed.ncb i.nlm.nih.g ov/16268861/ 